\title{
$M T H F R$ genotype and it's interaction with riboflavin in relation to blood pressure increase during normal pregnancy; preliminary findings from the OptiPREG project
}

\author{
E. O’Sullivan ${ }^{1}$, E. Psara ${ }^{1}$, K. Pentieva ${ }^{1}$, M. Ward ${ }^{1}$, G. Horigan $^{1}$, L. Doherty ${ }^{2}$, \\ K. Glackin ${ }^{3}$ and H. McNulty ${ }^{1}$ \\ ${ }^{1}$ Nutrition Innovation Centre for Food and Health, Ulster University, Cromore Road, Coleraine, \\ ${ }^{2}$ Causeway Hospital, The Northern Health and Social Care Trust, Coleraine and ${ }^{3}$ Altnagelvin Hospital, The Western \\ Health and Social Care Trust, Derry.
}

Hypertension in pregnancy (i.e. systolic/diastolic BP of 140/90 mmHg, as in non-pregnant adults) affects $10-15 \%$ of pregnancies and is of concern because it can lead to more serious hypertensive disorders in pregnancy ${ }^{(1)}$. The typical pattern of blood pressure (BP) in pregnancy is one of an initial small decrease until 16 weeks, followed by an increase to the end of pregnancy ${ }^{(2)}$. The common $677 \mathrm{C} \rightarrow \mathrm{T}$ polymorphism in the MTHFR gene is associated with an increased risk of hypertension in pregnancy ${ }^{(3)}$, however previous trials from this Centre in non-pregnant hypertensive adults have shown that riboflavin can lower BP specifically in those with the MTHFR 677TT genotype $^{(4)}$. In a previous opportunistic study based on BP retrospectively recovered from medical notes, we observed higher BP in women with the TT genotype during pregnancy ${ }^{(5)}$. Therefore, in the current study we hypothesised that pregnant women with the TT genotype will undergo a greater increase in BP during pregnancy and that this will be modulated by riboflavin status.

Data for this study were generated from the ongoing Optimal Nutrition for the Prevention of Hypertension (OptiPREG) project. Pregnant women were recruited at the $14^{\text {th }} \mathrm{GW}$ from antenatal clinics at Causeway hospital and the community in the Northern Trust, and Altnagelvin Hospital in the Western Trust in Northern Ireland. They were screened for the $M T H F R$ 677C $\rightarrow$ T genotype and BP measured according to the NICE guidelines. A blood sample was also collected to measure biomarker status of riboflavin using the erythrocyte gluthathione reductase activation coefficient (EGRac) assay.

Table 1. Systolic blood pressure at the $14 \mathrm{GW}$ and the change at $36 \mathrm{GW}$ by $M T H F R$ genotype and riboflavin status

\begin{tabular}{|c|c|c|c|c|c|c|c|c|c|c|c|c|}
\hline & \multicolumn{4}{|c|}{$\mathrm{CC}$} & \multicolumn{4}{|c|}{ CT } & \multicolumn{4}{|c|}{ TT } \\
\hline & \multicolumn{2}{|c|}{$14 \mathrm{GW}$} & \multicolumn{2}{|c|}{ Change* } & \multicolumn{2}{|c|}{$14 \mathrm{GW}$} & \multicolumn{2}{|c|}{ Change* } & \multicolumn{2}{|c|}{$14 \mathrm{GW}$} & \multicolumn{2}{|c|}{ Change* } \\
\hline & Mean & $\overline{\text { SEM }}$ & Mean & $\overline{\text { SEM }}$ & Mean & $\overline{\text { SEM }}$ & Mean & $\overline{\text { SEM }}$ & $\overline{\text { Mean }}$ & SEM & Mean & SEM \\
\hline Low' & $113 \cdot 0$ & $7 \cdot 7$ & $12 \cdot 3$ & $3 \cdot 8$ & $117 \cdot 5$ & $10 \cdot 4$ & $6 \cdot 4$ & $3 \cdot 1$ & $112 \cdot 2$ & $4 \cdot 5$ & $17 \cdot 8$ & $7 \cdot 7$ \\
\hline High & $118 \cdot 0$ & $7 \cdot 8$ & $8 \cdot 3$ & $3 \cdot 0$ & $120 \cdot 3$ & 8.9 & $2 \cdot 1$ & $2 \cdot 8$ & 114.4 & 4.9 & $4 \cdot 4$ & $7 \cdot 2$ \\
\hline
\end{tabular}

* Change is calculated on the difference between $\mathrm{BP}$ at $14 \mathrm{GW}$ and $36 \mathrm{GW}$.

Blood pressure change expressed as adjusted means and SEM, with adjustments for BMI and maternal age.

$\dagger$ 'Low' and 'high' refer to riboflavin categories were established by using the median value for the biomarker of riboflavin status, erythrocyte glutathione reductase activation coefficient (EGRac).

Abbreviations; GW gestational week.

These preliminary results suggest that women with the TT genotype and low riboflavin status undergo a markedly greater increase in systolic BP from the $14^{\text {th }}$ to the $36^{\text {th }} \mathrm{GW}$ (Table 1). A similar trend was also seen in diastolic BP but this did not reach significance. A randomised controlled trial is necessary to investigate the effect of riboflavin intervention on BP during pregnancy in women with the TT genotype; this is underway at our Centre with results from the trial expected in early 2019.

The authors gratefully acknowledge funding through an Enabling Award from the Northern Ireland HSC Public Health Agency, Research \& Development Division.

1. Bramham et al. (2014) BMJ 348, g2301.

2. Oostvogels et al. (2017) PloS One 19, e01775547.

3. Yang et al. (2014) PLoS ONE 9(2), e87497.

4. McNulty et al. (2017) Mol Aspects Med 53, 2-9.

5. O'Sullivan et al. (2017) Proc Nutr Soc 76(OCE3), E51. 